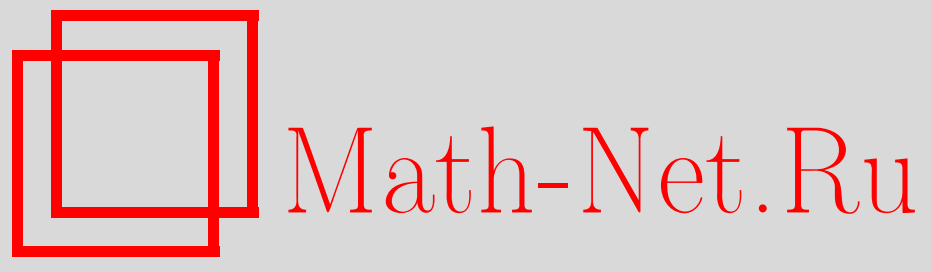

А. Ю. Плахов, О бильярдах в неограниченных областях, обращающих направление движения частиц, УМH, 2006, том 61, выпуск 1, 183-184

DOI: https://doi.org/10.4213/rm1699

Использование Общероссийского математического портала Math-Net.Ru подразумевает, что вы прочитали и согласны с пользовательским соглашением http://www . mathnet.ru/rus/agreement

Параметры загрузки:

IP : 3.95 .254 .165

26 апреля 2023 г., 16:58:31

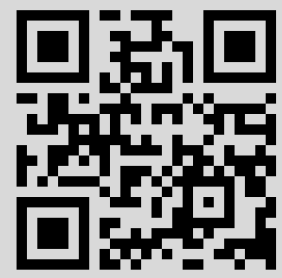




\title{
О бильярдах в неограниченных областях, обращающих направление движения частиц
}

\author{
А. Ю. Плахов
}

Рассмотрим евклидово пространство $\mathbb{R}^{2}$ с ортогональными координатами $x, y$ и бильярд в $\mathbb{R}^{2} \backslash \Omega$, где множество $\Omega$ удовлетворяет следующим условиям:

(а) $\Omega$ принадлежит верхней полуплоскости $\{y \geqslant 0\}$ и содержит полуплоскость $\{y>1\}$

(б) $\partial \Omega$ есть несамопересекающаяся кусочно-гладкая кривая класса $C^{2}$.

Обозначим $S_{+}^{1}=\left\{\vec{v}=\left(v_{1}, v_{2}\right) \in \mathbb{R}^{2}:|\vec{v}|=1, v_{2} \geqslant 0\right\}$ верхнюю единичную полуокружность и для произвольных $x \in \mathbb{R}, \vec{v} \in S_{+}^{1}$ рассмотрим бильярдную частицу, имеющую координаты $(x(t), y(t))=(x, 0)+\vec{v} t$ при $t \leqslant 0$. Если частица имеет конечное число отражений в регулярных точках $\partial \Omega$ и после этого движется свободно, то обозначим эту финальную скорость $\vec{v}_{\Omega}^{+}(x, \vec{v})$. Тем самым определена функция $\vec{v}_{\Omega}^{+}$на некотором подмножестве множества $\mathbb{R} \times S_{+}^{1}$, принимающая значения в $S_{-}^{1}:=\left\{\vec{v}=\left(v_{1}, v_{2}\right):|\vec{v}|=1, v_{2} \leqslant 0\right\}$. Справедливо следующее утверждение: функция $\vec{v}_{\Omega}^{+}$определена на открытом всюду плотном подмножестве множества $\mathbb{R} \times S_{+}^{1}$ и непрерывна. Доказательство этого утверждения несложно и в значительной своей части повторяет рассуждения, использовавшиеся в [1; лемма 1.7.1].

Основной результат настоящей работы - построение семейства множеств $\Omega_{\varepsilon}, \varepsilon>0$, удовлетворяющих условиям (а) и (б) и таких, что на любом борелевском подмножестве множества $\mathbb{R} \times S_{+}^{1}$ конечной лебеговской меры

$$
\text { при } \varepsilon \rightarrow 0^{+} \text {функция } \vec{v}_{\Omega_{\varepsilon}}^{+}(x, \vec{v}) \text { сходится по мере к }-\vec{v} \text {. }
$$

Построение заключается в следующем. Рассмотрим множество $E_{\varepsilon}=\{(x, y): y \geqslant 0$, $\left.y \geqslant \varepsilon(|x|-\varepsilon), x^{2} /\left(1+\varepsilon^{2}\right)+y^{2} \leqslant 1\right\}-$ фигуру, ограниченную сверху дугой эллипса, а снизу - большим диаметром и наклонными прямыми, проходящими через фокусы, и рассмотрим усеченный конус $K_{\varepsilon}=\{(x, y): y \geqslant 0, y \geqslant \varepsilon(x-\varepsilon), y \geqslant-\varepsilon(x+\varepsilon)\}$. Обозначим $E_{\varepsilon}^{k, b}, K_{\varepsilon}^{k, b}, k>0$, множества, полученные из $E_{\varepsilon}$ и $K_{\varepsilon}$ гомотетией с центром $(0,0)$ и коэффициентом $k$ и последующим сдвигом на вектор $(b, 0)$. Далее, определим по индукции множества $\mathscr{E}_{\varepsilon}^{(n)}$ и $\mathscr{K}_{\varepsilon}^{(n)}, n \in \mathbb{N}$. Положим $\mathscr{E}_{\varepsilon}^{(1)}=\bigcup_{m \in \mathbb{Z}} E_{\varepsilon}^{1,2 m \sqrt{1+\varepsilon^{2}}}$, $\mathscr{K}_{\varepsilon}^{(1)}=\bigcup_{m \in \mathbb{Z}} K_{\varepsilon}^{1,2 m \sqrt{1+\varepsilon^{2}}}$. Пусть теперь множества $\mathscr{E}_{\varepsilon}^{(n)}$ и $\mathscr{K}_{\varepsilon}^{(n)}$ уже определены для некоторого $n$. Рассмотрим пары $(k, b)$ такие, что расстояние между множествами $\mathscr{K}_{\varepsilon}^{(n)}$ и $E_{\varepsilon}^{k, b}$ равно $k$. (Под расстоянием между двумя множествами мы здесь понимаем нижнюю грань расстояний между элементами первого и элементами второго множества.) Множество всех таких пар счетно, они имеют одинаковую первую компоненту $k=k_{n}$, а множество вторых компонент $b=b_{n m}, m \in \mathbb{Z}$, образует бесконечную двустороннюю арифметическую прогрессию. Положим $\mathscr{E}_{\varepsilon}^{(n+1)}=\mathscr{E}_{\varepsilon}^{(n)} \cup\left(\bigcup_{m \in \mathbb{Z}} E_{\varepsilon}^{k_{n}, b_{n m}}\right)$, $\mathscr{K}_{\varepsilon}^{(n+1)}=\mathscr{K}_{\varepsilon}^{(n)} \cup\left(\bigcup_{m \in \mathbb{Z}} K_{\varepsilon}^{k_{n}, b_{n m}}\right)$. Множества $\mathscr{E}_{\varepsilon}^{(n)}$ инвариантны относительно сдвига на вектор $\left(2 \sqrt{1+\varepsilon^{2}}, 0\right)$, и каждое множество $I_{n}:=\left(\left[0,2 \sqrt{1+\varepsilon^{2}}\right] \times\{0\}\right) \backslash \mathscr{E}_{\varepsilon}^{(n)}$ есть объединение конечного числа интервалов, общая длина $\left|I_{n}\right|$ которых образует убывающую геометрическую прогрессию по $n$. Выберем $n_{\varepsilon}$ такое, что $\left|I_{n_{\varepsilon}}\right| \leqslant \varepsilon$, и положим $\Omega_{\varepsilon}=\{(x, y): y>0\} \backslash \mathscr{E}_{\varepsilon}^{\left(n_{\varepsilon}\right)}$. Очевидно, множество $\Omega_{\varepsilon}$ удовлетворяет условиям (а) и (б).

Обозначим $N_{\varepsilon}$ множество значений $(x, \vec{v}) \in \mathbb{R} \times S_{+}^{1}$ таких, что либо $v_{2} \leqslant \varepsilon / \sqrt{1+\varepsilon^{2}}$, либо $x+2 m \sqrt{1+\varepsilon^{2}} \in \bar{I}_{n_{\varepsilon}}$ для некоторого $m \in \mathbb{Z}$. Если $(x, \vec{v}) \notin N_{\varepsilon}$, то бильярдная частица в $\mathbb{R}^{2} \backslash \Omega_{\varepsilon}$, имеющая координаты $(x, 0)+\vec{v} t$ при $t \leqslant 0$, отразится ровно один раз в точке верхней границы одной из фигур $E_{\varepsilon}^{k_{n}, b_{n m}}$, и скорость после отражения равна $\vec{v}_{\Omega_{\varepsilon}}^{+}(x, \vec{v})=-\vec{v}+O(\varepsilon)$, причем эта оценка равномерна по $x$ и $\vec{v}$. С другой стороны, 
для любого борелевского множества конечной лебеговой меры $M \subset \mathbb{R} \times S_{+}^{1}$ лебегова мера пересечения $M \cap N_{\varepsilon}$ есть $O(\varepsilon)$. Таким образом, сходимость (1) доказана.

Это построение допускает обобщение на случаи размерности три и выше. В самом деле, рассмотрим евклидово пространство $\mathbb{R}^{3}$ с ортогональными координатами $x, y, z$; зафиксируем $\varepsilon>0$ и обозначим $\mathrm{O}_{\varepsilon}=\left\{(x, y, z): 0 \leqslant z \leqslant \sqrt{1-x^{2}-y^{2}}\right.$, $z \geqslant \varepsilon \max \{|x|-\varepsilon,|y|-\varepsilon\}\}$ множество, ограниченное сверху верхней единичной полусферой, а снизу - экваториальной плоскостью этой полусферы и четырьмя наклонными плоскостями. Обозначим $\mathrm{O}_{\varepsilon}^{k, x, y}, k>0$, множество, полученное из $\mathrm{O}_{\varepsilon}$ гомотетией с центром в начале координат и коэффициентом $k$ и последующим сдвигом на вектор $(x, y, 0)$. Подобно тому, как это было сделано в двумерном случае, строится множество $\mathscr{O}_{\varepsilon}$, являющееся объединением счетного набора попарно непересекающихся множеств вида $\mathrm{O}_{\varepsilon}^{k, x, y}$ (с, вообще говоря, различными тройками значений $k, x, y)$, инвариантное относительно сдвигов на векторы $(2,0,0)$ и $(0,2,0)$ и такое, что площадь плоского множества $([0,2] \times[0,2] \times\{0\}) \backslash \mathscr{O}_{\varepsilon}$ не превосходит $\varepsilon$. Множества $\Omega_{\varepsilon}^{(3)}=\{(x, y, z): z>0\} \backslash \mathscr{O}_{\varepsilon}$ содержатся в полупространстве $\{z \geqslant 0\}$, содержат полупространство $\{z>1\}$ и имеют кусочно-гладкую границу. Для почти всех $(x, y, \vec{v}) \in \mathbb{R}^{2} \times S_{+}^{2}$, где $S_{+}^{2}=\left\{\vec{v}=\left(v_{1}, v_{2}, v_{3}\right) \in \mathbb{R}^{3}:|\vec{v}|=1, v_{3} \geqslant 0\right\}$, бильярдная частица в $\mathbb{R}^{3} \backslash \Omega_{\varepsilon}^{(3)}$, имеющая координаты $(x, y, 0)+\vec{v} t$ при $t \leqslant 0$, испытает конечное число отражений и дальше движется свободно со скоростью $\vec{v}_{\Omega_{\varepsilon}^{(3)}}^{+}(x, y, \vec{v})$. Определенная таким образом функция $\vec{v}_{\Omega_{\varepsilon}^{(3)}}^{+}$сходится по мере к $-\vec{v}$ на любом подмножестве конечной лебеговой меры множества $\mathbb{R}^{2} \times S_{+}^{2}$.

Поясним смысл полученных результатов на следующем примере. На неограниченное тело в $\mathbb{R}^{2}$ падает вертикальный поток частиц плотности $\rho$ и фиксированного поперечного сечения $[a, b]$, т.е. первая конфигурационная координата падающих частиц заключена между $a$ и $b$. Тело в исходном положении совпадает с некоторым множеством $\Omega$, удовлетворяющим условиям (а) и (б). Тело может поворачиваться вокруг начала координат, причем вероятность поворота на угол $\varphi \in[-\pi / 2, \pi / 2]$ определяется вероятностной мерой $\nu$ на $S_{+}^{1}=\{(\cos \varphi, \sin \varphi), \varphi \in[-\pi / 2, \pi / 2]\}$. Математическое ожидание вертикальной составляющей силы давления потока на тело равно $R(\Omega)=\rho \int_{S_{+}^{1}} \int_{a}^{b}\left[1-\left(\vec{v}_{\Omega}^{+}\left(x / v_{2}, \vec{v}\right), \vec{v}\right)\right] d x d \nu(\vec{v})$. Требуется найти $\sup _{\Omega} R(\Omega)$.

Построенное выше семейство множеств $\left\{\Omega_{\varepsilon}\right\}$ доставляет решение этой задачи: именно, справедливо соотношение $\sup _{\Omega} R(\Omega)=2 \rho(b-a)=\lim _{\varepsilon \rightarrow 0^{+}} R\left(\Omega_{\varepsilon}\right)$. С другой стороны, математическое ожидание силы давления на вертикальную полуплоскость $\Omega^{0}:=\{(x, y): y>0\}$ равно $R\left(\Omega^{0}\right)=2 \rho(b-a) \int_{S_{+}^{1}} v_{2}^{2} d \nu(\vec{v}) ;$ в частности, если $\nu$ есть мера равномерного распределения на $S_{+}^{1}$, то величина $R\left(\Omega^{0}\right)=\rho(b-a)$ в 2 раза меньше максимального значения $R$.

В трехмерном случае аналогичное рассмотрение приводит к соотношению $\sup _{\Omega} R(\Omega)=2 \rho \sigma=\lim _{\varepsilon \rightarrow 0^{+}} R\left(\Omega_{\varepsilon}^{(3)}\right)$, где $\sigma-$ площадь поперечного сечения потока и $\rho$ - его плотность. $\mathrm{C}$ другой стороны, для верхнего полупространства $\Omega_{0}^{(3)}:=\{(x, y, z): z>0\}$ имеем $R\left(\Omega_{0}^{(3)}\right)=2 \rho \sigma \int_{S_{+}^{2}} v_{2}^{2} d \mu(\vec{v})$, где $\mu$ - вероятностная мера на $S_{+}^{2}$. В случае, если $\mu$ - мера равномерного распределения на $S_{+}^{2}$, величина $R\left(\Omega_{0}^{(3)}\right)=\frac{2}{3} \rho \sigma$ в 3 раза меньше максимального значения $R$.

В заключение выражаю благодарность А. М. Степину за полезные замечания.

\section{Список литературы}

[1] S. Tabachnikov, Billiards, Panor. Synthèses, 1, Société Mathématique de France, Paris, 1995.

А. Ю. Плахов (А. Yu. Plakhov)

Aveiro University, Portugal

E-mail: plakhov@mat.ua.pt
Представлено Д. В. Аносовым Принято редколлегией 01.12 .2005 